\title{
Geometry of Neighborhood Sequences in Hexagonal Grid ${ }^{\star}$
}

\author{
Benedek Nagy \\ GRLMC, Rovira i Virgili University, Tarragona, Spain \\ Faculty of Informatics, University of Debrecen, Hungary \\ H-4010 Debrecen P.O. Box 12 \\ nbenedek@inf .unideb.hu
}

\begin{abstract}
In this paper the nodes of the hexagonal grid are used as points. Three types of neighbors are used on this grid, therefore neighborhood sequences contain values 1,2 and 3. The grid is coordinatized by three coordinates in a symmetric way. Digital circles are classified based on digital distances using neighborhood sequences. They can be triangle, hexagon, enneagon and dodecagon. The corners of the convex hulls of these polygons are computed.
\end{abstract}

\section{Introduction}

The classical digital geometry started by [1], where the authors defined the two basic neighborhood relations on the square grid. The topic is well developed due to people of image processing and computer graphics communities. We refer to [2] as a recent textbook on the topic. In [3] the authors used the so-called neighborhood sequences to vary the neighborhood criterion in a path. They used only periodic neighborhood sequences in their analysis. Some properties of distances based on neighborhood sequences are detailed in [4. Nowadays, in many applications it is worth to consider other grids than the square one. The hexagonal grid has some nice properties and it is regular, therefore it is not too hard to handle it. The geometry of the hexagonal grid with a symmetric coordinate system is described in [5]. In [6] the neighborhood sequences were also defined for the hexagonal grid. In this paper we will analyse some properties of the distances based on neighborhood sequences on this grid.

The structure of the paper is as follows. In the second section we give our notation, and provide some properties of the concepts introduced. In the other sections we detail some former results of Das and Chatterji [4] on the hexagonal grid. We use only initial parts of the neighborhood sequences in our analysis, therefore we do not care about the periodic property of the whole neighborhood sequences. In the third section we describe the smallest digital circles of the hexagonal grid using only a step from the origin. In the fourth section changing and developments of wave-fronts and digital circles are analysed. We compute

\footnotetext{
* This research was supported by grants from the Hungarian Foundation for Scientific Research (OTKA F043090 and T049409).
} 
the coordinate values of the corners of these polygons. In the fifth section we give a description of all the digital circles with neighborhood sequences in the hexagonal grid. We show a characterization of them. We present some properties, in which the hexagonal grid differs from the square grid. In the last section we summarize our results.

\section{Basic Notation and Concepts}

In this section we recall some definitions and notation from the literature mentioned earlier concerning neighborhood relations and sequences.

There are usually three types of neighbors defined, as Fig. 1 shows, among the nodes of the hexagonal grid.

In Figure 1 a node and its 12 neighbors are shown. Only the 1-neighbors are directly connected by an edge, the other 2- and 3-neighbors are at the positions of shorter and longer diagonals, respectively. These relations are reflexive (i.e., a node is a 1-, 2-, and a 3-neighbor of itself by definition) and symmetric. In

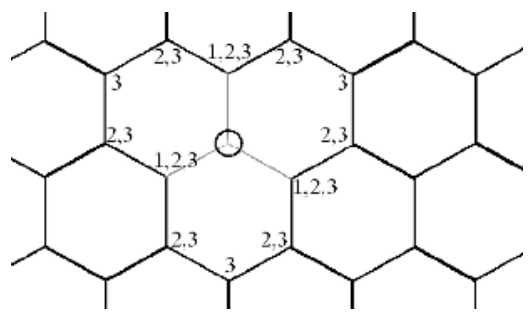

Fig. 1. Types of neighbors in the hexagonal grid

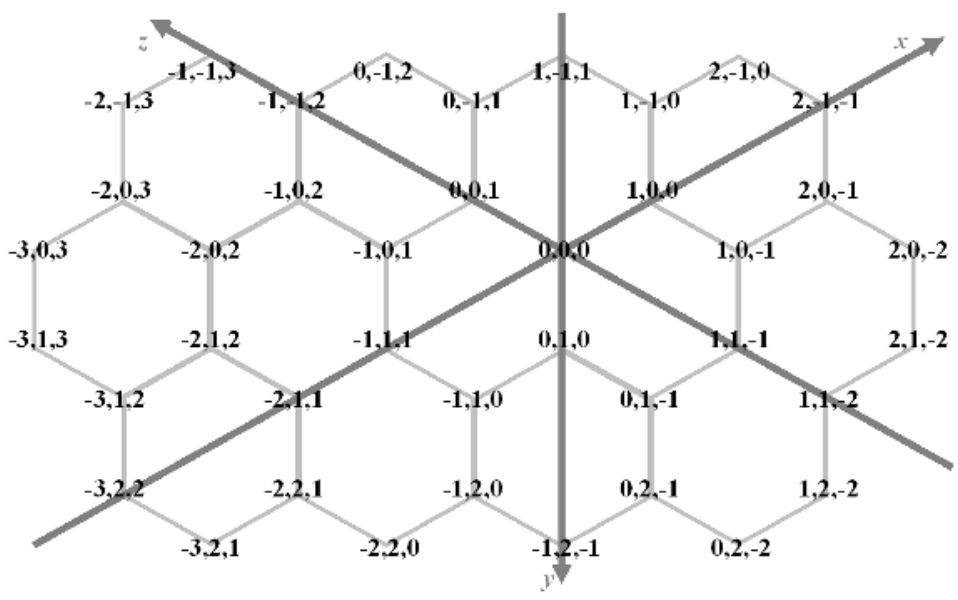

Fig. 2. Coordinate values of nodes 
addition, all 1-neighbors of a point are its 2-neighbors and all 2-neighbors are 3 -neighbors, as well (i.e., they have increasing and inclusion properties).

The coordinate values of the grid were introduced as it is shown in Fig. 2, The coordinate axes meet at a grid-point called Origin having triplet $(0,0,0)$. They are the direction of grid-edges starting at the Origin. The coordinate values of each point can be computed as the sum of steps on the grid-edges taken into direction of the edges. A step by direction of axis $x$ increases the first coordinate value by 1, while a step to inverse direction decreases the first coordinate. Similarly steps on the edges parallel to axis $y$ and $z$ modify the second and third coordinate values, respectively. Three coordinate values are used to address a point taking advantage of the symmetry of the grid.

With the help of the assigned coordinate values we are able to describe the grid in a mathematical way. By the presented coordinate system every node has a unique triplet which exactly shows the place of the node. The hexagonal grid contains exactly those triplets which have sum of coordinate values 0 or 1 . We call the points with 0 -sum value even (their connections have shape $\mathbf{Y}$ in the figure); the points with 1-sum are odd (opposite shape). We can write the neighborhood relations in the following formal form.

The points $P(p(1), p(2), p(3))$ and $Q(q(1), q(2), q(3))$ of the hexagonal grid are $m$-neighbors $(m=1,2,3)$, if the following two conditions hold:

1. $|p(i)-q(i)| \leq 1$, for $i=1,2,3$,

2. $|p(1)-q(1)|+|p(2)-q(2)|+|p(3)-q(3)| \leq m$.

It is easy to check that the formal definition above with the presented coordinate values (Fig. 2) gives the neighborhood relations shown in Fig. 1

Now, we are recalling some concepts about the theory of neighborhood sequences. In this paper, we are dealing only with neighborhood sequences in this grid. The sequence $B=(b(i))_{i=1}^{\infty}$, where $1 \leq b(i) \leq 3$ for all $i \in \mathbb{N}$, is called a neighborhood sequence (on the hexagonal grid). When we need only the initial part up to the $l$-th element, then we briefly write $B_{l}=(b(1), b(2), \ldots, b(l))$.

A movement is called a $b(i)$-step when we move from a point $P$ to a point $Q$ and they are $b(i)$-neighbors. Let $P, Q$ be two points and $B$ be a neighborhood sequence. The point-sequence $P=P_{0}, P_{1}, \ldots, P_{k}=Q$, in which we move from $P_{i-1}$ to $P_{i}$ by a $b(i)$-step $(1 \leq i \leq k)$, is called a $B$-path from $P$ to $Q$ of length $k$. The $B$-distance $d(P, Q ; B)$ from $P$ to $Q$ is defined as the length of the shortest $B$-path(s). In a $B$-path an initial sequence of $B$ is used.

The sequence of 1-neighbor points, for which a coordinate value remains constant, forms a so-called lane. In Fig. 3 there are some examples; the black line shows the lane for which the third coordinate is 0 and the gray lane represents the lane for which the second value is 0 .

Every lane is 'orthogonal' to one of the coordinate axes, especially that one of $\{x, y, z\}$, for which the coordinate value is fixed.

If a point is on an axis, then two of its coordinate-values coincide. For instance on axis $x$ they are the second and third ones (meaning that the point is on the 


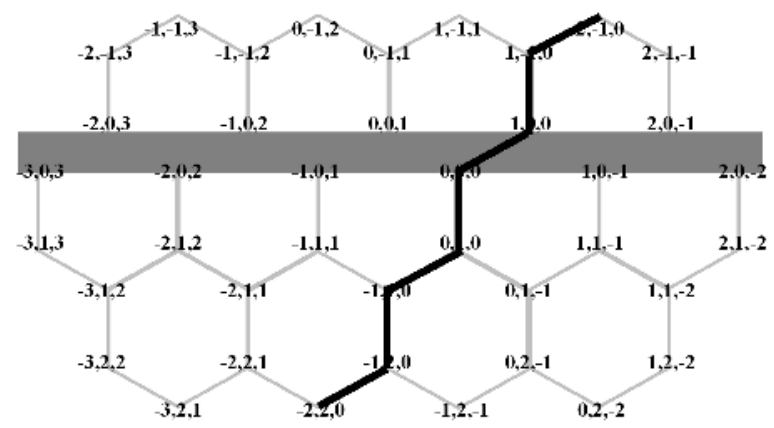

Fig. 3. Examples for lanes

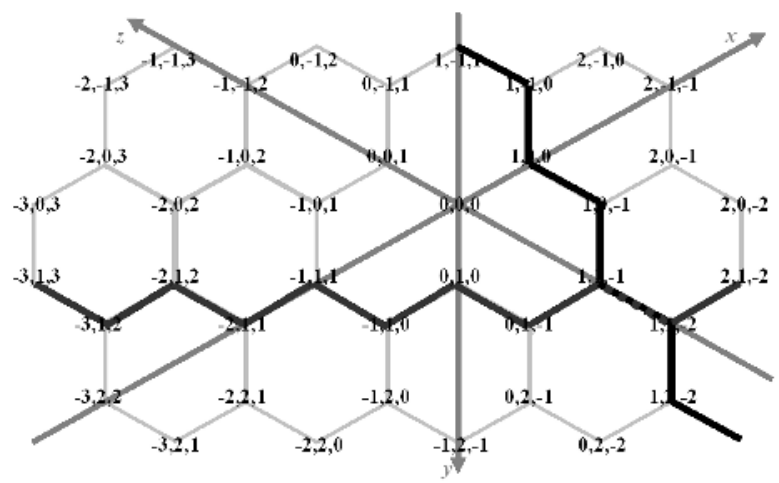

Fig. 4. Lanes and coordinate axes

lanes for which the same value is fixed on axes $y$ and $z$ ). See Fig. 4, where the lanes $x=1$ and $y=1$ are shown with black and dark-gray, respectively. They are orthogonal to the axes $x$ and $y$, respectively. The two points where they meet are on the axis $z$. It is a nice property of the assignment of coordinates to the grid, that a point and its symmetric pair mirroring it to an axis have the same coordinate values, but - if they are not on the symmetry axis, - in a different order. So a point and its mirror images are identical up to a permutation of their coordinates.

To use the line between the lanes $x=0$ and $x=1$ as a symmetry axis such that these two lanes are mirror images of each others we have the following formula. The mirror image of the point $P(x, y, z)$ is given as $P^{\prime}(-(x-1),-z,-y)$.

In this paper we investigate the way of a neighborhood sequence spreads in the digital space starting from a point of the hexagonal grid. This spreading is translation-invariant among the points of the same parity and it is symmetric concerning points with different parities. So, for simplicity we may choose the Origin $\mathbf{O}(0,0,0)$ as the starting point. 
Let

$$
C_{B_{k}}=\{P: d(\mathbf{O}, P ; B) \leq k\} .
$$

It is the region (digital circle) occupied by $B$ after the first $k$ steps.

In 4] Das and Chatterji showed that for every initial part of a neighborhood sequence in the square grid (using Cartesian coordinates and two types of neighbors), the obtained digital circle is always an octagon. These octagons can be degenerated, so the digital circles are squares in the following cases: Using only 1-steps in the initial part of the neighborhood sequence we get only four edges (the corners will be $(0, k),(k, 0),(0,-k),(-k, 0))$, while using only 2 -steps we get a square with corners $(k, k),(k,-k),(-k,-k),(-k, k)$. In the case when we use both 1-step(s) and 2-step(s) our result is a non-degenerated octagon.

The following observations hold in the square grid, and they are true in the hexagonal case as well:

Remark 1. The convex hull of every $C_{B_{k}}$ is digitally convex in the usual sense (see e.g. p. 171, Definition 4.3.4. in [7])

On figures we will use the convex hull as the occupied polygon of the digital circle. These polygons has sides and corners in the usual sense. Since they are convex, the sets of the coordinate triplets of corners describe them. (We use the term corner only for angles less than $\pi$.)

Remark 2. For any neighborhood sequence $B$, the sequence of regions $\left(C_{B_{k}}\right)_{k=1}^{\infty}$ is a strictly monotone increasing sequence. That is, $k>l$ implies $C_{B_{k}} \supsetneq C_{B_{l}}$.

In the following sections we will underline some properties which are different for the digital circles in square grid and in hexagonal grid. Now, we are moving to describe all digital circles of the hexagonal grid in details.

\section{Description of Small Circles}

Table 1 shows the three possible circles obtained by a step.

The three circles have four kinds of corners. Corner-type $\alpha$ signs corners with angle $\frac{\pi}{3}$. Corner-types $\beta$ and $\gamma$ refer for angles $\frac{2 \pi}{3}$ (where the sides connected at a corner type $\gamma$ are parallel to some edges and so to coordinate axes of the hexagonal grid, and the sides at a corner type $\beta$ are orthogonal to some edges and so to coordinate axes of the grid.) At corners type $\delta$ the angles are $\frac{5 \pi}{6}$. Note that the circle obtained by a 2-step from $\mathbf{O}$ is the same as the circle obtained by two 1-steps. This property (a circle can have more radii, depending on the used neighborhood sequences) is not present in the square grid.

Proposition 1. Contrary to the square grid in the hexagonal grid it is possible for two neighborhood sequences $B^{\prime}, B^{\prime \prime}$ that $C_{B_{k}^{\prime}}=C_{B_{l}^{\prime \prime}}$ with $k \neq l$.

Let $B^{\prime}$ be given such a way that $B_{2}^{\prime}=(1,1)$ and let it hold for $B^{\prime \prime}$ that $B_{1}^{\prime \prime}=(2)$ then $C_{(1,1)}=C_{(2)}$. 
Table 1. Digital circles with radius 1 by various steps

\begin{tabular}{|c|c|c|}
\hline $\begin{array}{l}\text { Initial part of the } \\
\text { neighbourhood sequence }\end{array}$ & The shape of the circle (figure) & Corner-types and coordinates \\
\hline 1 & & $\begin{array}{l}\text { triangle with number } 3 \alpha \text {-type: } \\
\alpha(1,0,0) \\
\alpha(0,1,0) \\
\alpha(0,0,1)\end{array}$ \\
\hline$C_{(1)}$ & & \\
\hline $\begin{array}{l}11 \\
2 \\
C_{(2)}^{\prime}=C_{(1,1)}\end{array}$ & & $\begin{array}{l}\text { hexagon with number } 6 \beta \text {-type: } \\
\beta(1,-1,0) \\
\beta(1,0,-1) \\
\beta(0,1,-1) \\
\beta(0,-1,1) \\
\beta(-1,1,0) \\
\beta(-1,0,1)\end{array}$ \\
\hline $\begin{array}{l}3 \\
\\
C_{(3)}\end{array}$ & & $\begin{array}{l}\text { enneagon with } \\
\gamma(1,-1,0) \\
\gamma(1,0,-1) \\
\gamma(0,1,-1) \\
\gamma(0,-1,1) \\
\gamma(-1,1,0) \\
\gamma(-1,0,1) \\
\delta(1,1,-1) \\
\delta(1,-1,1) \\
\delta(-1,1,-1)\end{array}$ \\
\hline
\end{tabular}

\section{Development of Corners}

In 3] the authors analysed the wavefront sets of neighborhood sequences in the square grid. In this part we are detailing how the wave-fronts are occupying the hexagonal grid. Because of symmetry it is sufficient to deal with a sixth of the plane. For our convenience we will use the region between the axes $z$ and $y$, especially between the positive part of $z$ and the negative part of $y$ (we refer here to Fig. 2). One can check that the points $P(x, y, z)$ with $z \geq x \geq y$ are exactly those ones which are in this sixth of the plane. We will call this region as the analysed section.

It is sufficient to deal with this analysed section since we have the following statements. For any point $P$ the points obtained by permutating the coordinatevalues of $P$ are exactly the same points as the mirror images of $P$ obtained by mirroring it to some of the coordinate axes. Every point has the same parity as its symmetric pairs. Let $P$ be given as $(p(1), p(2), p(3))$. It is easy to check that, for instance, the mirror image of $P$ with axis $x$ is $P^{\prime}(p(1), p(3), p(2))$. Similar facts hold for the other mirror points. A point with its mirror images represent 
at most six points. In this case there are not equal coordinate values, i.e. the point is not on an axis. Every point has a mirror image in the analysed section of the grid (using only axial mirroring to the coordinate axes). It can be obtained by permutation of coordinate-values.

Let us check which corners of the small circles are in the analysed section. $C_{(1)}$ has only one corner in this section, namely $\alpha(0,0,1) . C_{(2)}$ has also only one corner in this area of the grid, namely $\beta(0,-1,1)$, while $C_{(3)}$ has two corners in the analysed section; they are $\gamma(1,-1,1)$ on axis $y$ and $\delta(0,-1,1)$ with an orthogonal edge to axis $z$.

Now we analyze how the possible vertices change in the growing steps. Table 2 lists all kinds of corners occur in different digital circles. (We detail only the changing way of the coordinate values of those corners which are in the analysed sixth of the hexagonal plane.) The table shows all possibilities of the evolution of the corners by a step in the analysed section of the grid. It is due to the facts that all corners of Table1 (which are in the analysed section) are also in Table2, and all possible evolving corners are in Table 2, as well.

Based on Table 2 we summarize the evolution of corners via the growing procedure.

\section{Proposition 2}

- (a) The new corner(s) are b-neighbors of the previous one when we obtain them by a b-step.

- (b) A corner type $\alpha$ occurs only at digital circle $C_{(1)}$.

- (c) A corner type $\gamma$ occurs only when two coordinate values have the same value, i.e. the corner is on an axis.

- (d) In some steps a corner type $\beta$ or a corner type $\gamma$ is divided to two corners type $\delta$.

- (e) If a corner type $\gamma$ remains the same type after a step then it stays on the same axis as before (by changing the parity).

- (f) The corners type $\delta$ never change their type, moreover their position is fixed, i.e. when a side of the corner was orthogonal to an axis then this is also the case after any kind of step.

- (g) The corners type $\delta$ coming from a corner type $\gamma$ have a side orthogonal to that axis on which the corner $\gamma$ was.

- (h) In some cases the resulted digital object by a 3-step is the same as the one obtained by a 2-step.

Proof. Most of the statements above are easy to check. We analyse only the statements (e) and (f). Let us start with (e).

If a corner $\gamma(x, y, z)$ on the axis $y$, then $x=z$. If it is even then with a 3 -step we get $\gamma(x+1, y-1, z+1)$ which is also on the axis $y$. Similarly if $\gamma$ is odd then with a 1-step the first and the third coordinate values do not change, therefore the new corner is on the axis $y$ as well. The same analysis works when $\gamma$ is on the axis $z$. It is evident that the parity of the corner is changing by these steps.

To prove (f) assume that $\delta(x, y, z)$ has an edge which is orthogonal to the axis $y$. Then the mirror images of $\delta$ are also corners. Let $\delta^{\prime}(z, y, x)$ be its 
Table 2. Development table of corners by taking a step

\begin{tabular}{|c|c|c|c|}
\hline $\begin{array}{l}\text { original corner: type } \\
\text { and coordinates }\end{array}$ & $\begin{array}{l}\text { corner after a } \\
\text { 1-step }\end{array}$ & $\begin{array}{l}\text { corner after a 2- } \\
\text { step }\end{array}$ & corner after a 3 -step \\
\hline$\alpha(0,0,1)$ & $\beta(0,-1,1)$ & $\beta(0,-1,2)$ & $\begin{array}{l}\gamma(-1,-1,2) \text { on axis } z \\
\delta(0,-1,2) \text { with edge or- } \\
\text { thogonal to axis } y\end{array}$ \\
\hline$\beta(x, y, z)$ even & $\beta(x, y, z+1)$ & $\beta(x, y-1, z+1)$ & $\begin{array}{l}\delta(x, y-1, z+1) \text { with edge } \\
\text { orthogonal to axis } z, \\
\delta(x+1, y-1, z+1) \text { with } \\
\text { edge orthogonal to axis } z\end{array}$ \\
\hline$\beta(x, y, z)$ odd & $\beta(x, y-1, z)$ & $\beta(x, y-1, z+1)$ & $\begin{array}{l}\delta(x, y-1, z+1) \text { with edge } \\
\text { orthogonal to axis } y \\
\delta(x-1, y-1, z+1) \text { with } \\
\text { edge orthogonal to axis } z\end{array}$ \\
\hline $\begin{array}{|lll|}\gamma(x, y, z) & \text { even } & \text { on } \\
\text { axis } y & & \\
\end{array}$ & $\begin{array}{l}\delta(x, y, z+1) \\
\delta(x+1, y, z)\end{array}$ & $\begin{array}{l}\delta(x, y-1, z+1) \\
\delta(x+1, y-1, z)\end{array}$ & $\gamma(x+1, y-1, z+1)$ \\
\hline $\begin{array}{|lll|}\gamma(x, y, z) & \text { odd } & \text { on } \\
\text { axis } y & & \\
\end{array}$ & $\gamma(x, y-1, z)$ & $\begin{array}{l}\delta(x, y-1, z+1) \\
\delta(x+1, y-1, z)\end{array}$ & $\begin{array}{l}\delta(x, y-1, z+1) \\
\delta(x+1, y-1, z)\end{array}$ \\
\hline \begin{tabular}{lll|}
$\gamma(x, y, z)$ & even & on \\
axis $z$ & & \\
\end{tabular} & $\gamma(x, y, z+1)$ & $\begin{array}{l}\delta(x-1, y, z+1) \\
\delta(x, y-1, z+1)\end{array}$ & $\begin{array}{l}\delta(x-1, y, z+1) \\
\delta(x, y-1, z+1)\end{array}$ \\
\hline $\begin{array}{|lll|}\gamma(x, y, z) & \text { odd } & \text { on } \\
\text { axis } z & & \\
\end{array}$ & $\begin{array}{l}\delta(x-1, y, z) \\
\delta(x, y-1, z)\end{array}$ & $\begin{array}{l}\delta(x-1, y, z+1) \\
\delta(x, y-1, z+1)\end{array}$ & $\gamma(x-1, y-1, z+1)$ \\
\hline $\begin{array}{l}\delta(x, y, z) \text { even with } \\
\text { edge orthogonal to } \\
\text { axis } y\end{array}$ & $\delta(x, y, z+1)$ & $\delta(x, y-1, z+1)$ & $\delta(x+1, y-1, z+1)$ \\
\hline $\begin{array}{l}\delta(x, y, z) \text { odd with } \\
\text { edge orthogonal to } \\
\text { axis } y\end{array}$ & $\delta(x, y-1, z)$ & $\delta(x, y-1, z+1)$ & $\delta(x, y-1, z+1)$ \\
\hline $\begin{array}{l}\delta(x, y, z) \text { even with } \\
\text { edge orthogonal to } \\
\text { axis } z\end{array}$ & $\delta(x, y, z+1)$ & $\delta(x, y-1, z+1)$ & $\delta(x, y-1, z+1)$ \\
\hline $\begin{array}{l}\delta(x, y, z) \text { odd with } \\
\text { edge orthogonal to } \\
\text { axis } z\end{array}$ & $\delta(x, y-1, z)$ & $\delta(x, y-1, z+1)$ & $\delta(x-1, y-1, z+1)$ \\
\hline
\end{tabular}

symmetric pair to axis $y$. Then the side of the polygon connecting $\delta$ and $\delta^{\prime}$ is orthogonal to the axis $y$. After any kind of step the new corners obtained from $\delta$ and $\delta^{\prime}$ have the same property. The proof is similar if one of the edges connected at corner $\delta$ is orthogonal to axis $z$.

As we can see the corner-types are in a closed set, i.e. we cannot step out from the above used set by the growing steps.

Now, we present a method which calculates the corners of any digital circle. We are using Table 1 and the transition table given in Table 2.

Let us calculate the corner of the digital circle with origin $\left(x_{0}, y_{0}, z_{0}\right)$ using the initial part $B_{k}$ of a neighborhood sequence $B$. First we compute the corners of $C_{B_{k}}$ using $\mathbf{O}$ as origin, and after this we will translate the circle. 


\section{Algorithm}

1. Initially start with corners of the circle $C_{(b(1))}$ from Table 1 which are in the analysed section of the grid, where $b(1)$ is the first element of $B$. Let $i=1$.

2. While $i<k$ let $i:=i+1$ and the analysed corners of the new polygon are from Table 2 by a $b(i)$-step. (Keep only those ones which are in the analysed part of the grid.)

3. Get every point which coordinate values form a permutation of a computed point. Those are the corners of $C_{B_{k}}$.

4. If $\left(x_{0}, y_{0}, z_{0}\right)$ is even then add the vector $\left(x_{0}, y_{0}, z_{0}\right)$ to each corner of $C_{B_{k}}$ in order to get the result.

5. If $\left(x_{0}, y_{0}, z_{0}\right)$ is odd then then, first, let the new values of coordinates of the corners be given by the formula $(x, y, z) \rightarrow(-(x-1),-z,-y)$ (they represent the digital circle with origin $(1,0,0)$ generated by $B$ with radius $k$ );

secondly, add the vector $\left(x_{0}-1, y_{0}, z_{0}\right)$ to each corner to obtain the final result.

Now we present an example. Let us determine the corners of the digital circle starting from point $(-5,3,3)$ with $B_{4}=(1,3,1,2)$.

1. $b(1)=1$, therefore we start with $C_{(1)}$, so we have $\alpha(0,0,1)$ and $i=1$.

2. $i<4$ therefore $i:=2, b(i)=b(2)=3$, the result: $\gamma(-1,-1,2)$ and $\delta(0,-1,2)$. $i<4$ therefore $i:=3, b(i)=b(3)=1, \gamma(-1,-1,2)$ is even and it is on axis $z$ (the first two coordinates have the same value): $\gamma(-1,-1,3)$ and $\delta(0,-1,2)$ is odd and it has edge orthogonal to the axis $y$ so the new corner: $\delta(0,-2,2)$.

$i<4$ therefore $i:=4, b(i)=b(4)=2, \gamma(-1,-1,3)$ is odd and it is on axis $z$, so we get $\delta(-2,-1,4)$ and $\delta(-1,-2,4)$, but the first one is outside of the analysed section of the plane

$\delta(0,-2,2)$ is even and it has edge orthogonal to the axis $y$ so the new corner: $\delta(0,-3,3)$.

$i=4$, the loop of Step 2 is finished.

3. The corners with permutations: all of them are type- $\delta$ : $(-1,-2,4)$, $(-2,-1,4),(-1,4,-2),(-2,4,-1),(4,-1,-2),(4,-2,-1)$ and $(0,-3,3)$, $(0,3,-3),(-3,0,3),(-3,3,0),(3,0,-3),(3,-3,0)$.

4. The given origin is odd, so

5. first we get: $(2,-4,2),(3,-4,1),(2,2,-4),(3,1,-4),(-3,2,1),(-3,1,2)$, $(1,-3,3),(1,3,-3),(4,-3,0),(4,0,-3),(-2,3,0),(-2,0,3)$.

Secondly, adding $(-6,3,3)$ the final result: $(-4,-1,5),(-3,-1,4)$, $(-4,5,-1),(-3,4,-1),(-9,5,4),(-9,4,5),(-5,0,6),(-5,6,0),(-2,0,3)$, $(-2,3,0),(-8,6,3),(-8,3,6)$ and all of them are type- $\delta$.

Using the three digital circles with radius 1 and our growing table we get all possible digital circles of the hexagonal grid. In the next section we will list their types. 


\section{The Shapes of the Digital Circles}

In this section - continuing our previous results - we characterize the digital circles by neighborhood sequences in the hexagonal grid.

Theorem 1. The shape of the digital circle generated by the neighborhood sequence $B$ in $k$ steps is a triangle if and only if it is $C_{(1)}$. The shape is a hexagon if and only if there is no element 3 in the initial part of $B$ up to the $k$-th element. (Except the previous case, in which $C_{(1)}$ is especially a triangle.) The shape is an enneagon if and only if there is not any element 2 and nor any consecutive 1,1 or 3,3 occur in the initial part $B_{k} .\left(\right.$ Except $C_{(1)}$.) In every other case the digital circle is a dodecagon.

Proof. In the first case the triangle has three $\alpha$ corners. According to point (b) of Proposition 2 only other types of corners can occur at the other digital circles. With other types of corners it is impossible to get a triangle.

Now let us consider the other digital circles. It is easy to check in Table 1 that starting with an element 2 we get a hexagon with six $\beta$ corners. Moreover by Table 2 we know that using 1-step and/or 2-step from corners type $\alpha$ and from corners type $\beta$ the new corners will be type $\beta$ as well. With only corners type $\beta$ there must be six of them to make a polygon. Therefore without a 3 -step the result is a hexagon with corners type $\beta$.

It is shown in Table 1 that $C_{(3)}$ is an enneagon with three $\gamma$ and $\operatorname{six} \delta$ corners. The corner $\gamma(1,-1,1)$ is odd and it is on the axis $y$. Therefore with a 1-step it grows to a $\gamma_{2}$ which is even and is on the same axis. (With a 2-step or a 3 -step the corner would be divided to two $\delta$ vertices.) From $\gamma_{2}$ with a 3 -step $\gamma_{3}$ is resulted; it is odd on axis $y$. (From $\gamma_{2}$ with a 1-step or a 2-step we would obtain two $\delta$ corners.) Since $\gamma_{3}$ is in the same 'class' (i.e. in the same row of Table 2) as $\gamma(1,-1,1)$ the computing cycle is starting again, and it is going on while the steps are 1-step and 3-step turn by turn. Observe in Table 2 that type $\gamma$ corner can be obtained from $\alpha$, but cannot from $\beta$. Therefore there is no way to get $\gamma$ vertices from $C_{(2)}$ and so from any hexagons. From $C_{(1)}$ one can obtain an enneagon in one way, namely to get $C_{(1,3)}$. The obtained corner $\gamma(-1,-1,2)$ is even and it is on the axis $z$. One can check that there is a similar computing cycle for this $\gamma$ to keep it with only 1-steps and 3-steps by turns. (Leaving this computing cycle two corners type $\delta$ are obtained instead of the type $\gamma$.)

Finally, in all other cases the polygons only have $\delta$ vertices. When twelve corners type $\delta$ are in a digital circle, then it never happens that they change to another type (see Table 2) and twelve of them are needed for a polygon. Therefore the last statement is proved.

Analysing the digital circles on the square and on the hexagonal grid we have found the following important difference.

In the square grid the region occupied by $k$ steps of a neighborhood sequence $A$ is independent of the ordering of the first $k$ element of $A$. (see [4]) 


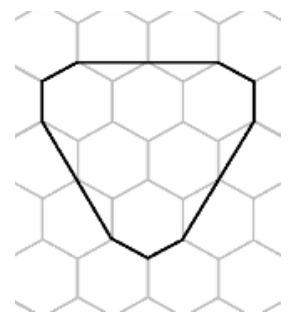

(a) Digital circle by $(1,3)$

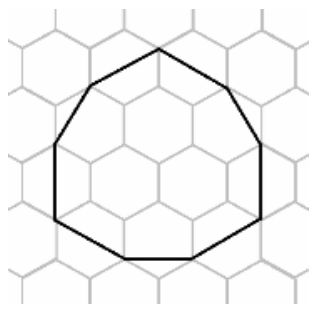

(b) Digital circle by $(3,1)$

Fig. 5. The elements of the neighborhood sequence on the hexagonal grid are not permutable, in general

Proposition 3. Contrary to the case of the square grid, it is possible for a neighborhood sequence $B$ and for a $k \in \mathbb{N}$, that the region $C_{B_{k}}$ depends on the order of the first $k$ elements of $B$.

Assume that $B_{2}^{\prime}=(1,3)$ and $B_{2}^{\prime \prime}=(3,1)$ then our regions $C_{B_{2}^{\prime}}$ and $C_{B_{2}^{\prime \prime}}$ differ as Fig. [5] shows.

\section{Conclusions}

In this paper we presented some results about neighborhood sequences on the hexagonal grid. We made a classification of the digital circles. We gave the possible types of corners of these digital polygons and studied their development in the growing procedure. Moreover an algorithm is presented to compute the coordinate-values of the corners of any digital circle (arbitrary origin, arbitrary neighborhood sequence and arbitrary radius) on the hexagonal grid. We listed the types of the digital circles occupied by neighborhood sequences, as well. Since the convex hulls of the digital circles are convex polygons, the lists of their corners determine them.

Our results can be used in digital image processing and in the field of networks as well. It is useful in region growing procedures. In grid-structured networks the non-common properties are useful. Some digital circles have several radii or the non permutability of the elements of the neighborhood sequence are exotic properties. In practice, it would also be interesting to analyse the development of the wave-front sets in the case of "barrels", or starting not from a point, but from other digital object, for instance from a lane. Another possible direction of future research is the further analysis of meeting waves, etc. It would be interesting if one mixed our method of region growing with the methods used in practice ( $8,9,2])$. Extensions to non-regular grids can be topics of further research, as well.

\section{References}

1. Rosenfeld, A., Pfaltz, J.L.: Distance functions on digital pictures. Pattern Recognition 1 (1968) 33-61

2. Klette, R., Rosenfeld, A.: Digital geometry. Geometric methods for digital picture analysis. Morgan Kaufmann Publishers, San Francisco, CA, Elsevier Science B.V., Amsterdam (2004) 
3. Das, P.P., Chakrabarti P.P., Chatterji, B.N.: Distance functions in digital geometry. Inform. Sci. 42 (1987) 113-136

4. Das, P.P., Chatterji, B.N.: Octagonal distances for digital pictures. Inform. Sci. 50 (1990) 123-150

5. Nagy, B.: A symmetric coordinate system for the hexagonal networks. In: Proceedings of Information Society 2004 - Theoretical Computer Science, ACM conference, Ljubljana, Slovenia (2004) 193-196

6. Nagy, B.: Shortest path in triangular grids with neighbourhood sequences. Journal of Comp. and Inf. Techn. 11 (2003) 111-122

7. Voss, K.: Discrete images, objects, and functions in $\mathbb{Z}^{n}$. Algorithms and Combinatorics Vol. 11. Springer-Verlag, Berlin Heidelberg New York (1993)

8. Chen, C.H., Pau, L.F., Wang, P.S.P. (eds.): Handbook of pattern recognition \& computer vision. Handbooks in Science and Technology. Academic Press, Orlando, FL (1986)

9. Gonzalez, R.C., Woods, R.E.: Digital image processing. Addison-Wesley, Reading, MA (1992) 